\title{
Einleitung
}

Corina L. Petrescu, Alison Lewis, Valentina Glajar

Geschichten der Gewalt hinterlassen Spuren nicht nur in den einzelnen Personen, die ihnen ausgeliefert sind, sondern auch in Menschengruppen, die mit den Ereignissen und Folgen von Gewalt zurechtkommen müssen. Umso schwerer ist die Verarbeitung dieser Handlungen, wenn sowohl die Täter als auch die Opfer derselben Gruppe angehören - sei es derselben nationalen, sozialen oder ethnischen Gruppierung. Dadurch wird nicht nur die Möglichkeit einer Distanzierung von den Gewalttaten, sondern auch das kollektive Erinnern daran erschwert.

Die politischen Regime des ehemaligen Ostblocks während des Kalten Krieges (1947-1991) waren Gewaltherrschaften. Sie kamen überwiegend mit Hilfe der sowjetischen Armee an die Macht, die in ihren Anfangsjahren auch dafür sorgte, dass ihnen diese Macht erhalten blieb, auch wenn es teilweise gegen den Wunsch und Willen der Bevölkerung war. Neben den Vertretern der jeweiligen kommunistischen Parteien existierten innerhalb dieser Regime die Geheimdienste, die gleichzeitig auch eine politische Polizei und ein Herrschaftsinstrument nach sowjetischem Vorbild waren. Die Aufgabe dieser staatspolizeilichen Organe war es, die Partei vor Angriffen des ,Feindes ‘ zu schützen, die sowohl von außen als auch von innen kommen mochten. Die Geheimdienste des Ostblocks nahmen vor allem ihre eigene Bevölkerung ins Visier und scheuten nicht davor zurück, sie durch physische und psychische Gewalt zum Gehorsam zu zwingen. Zur Überwachung und Disziplinierung der eigenen Bevölkerung wurden vorzugsweise Personen aus dem gleichen sozialen oder beruflichen Milieu als Informanten oder Kontaktpersonen herbeigezogen, die unauffällig und lautlos die Repression durchsetzen sollten. Unterdrücker und Unterdrückte, Täter und Opfer lebten und wirkten zusammen in einem Netz von Beziehungen, dessen Komplexität erst nach dem Fall der Regime bekannt wurde und immer noch erklärungsbedürftig ist. Dieses Themenheft nimmt sich eine Annäherung an diese Zeit und die problematischen Beziehungen zwischen den post-kommunistischen Nachfolgegesell- 
schaften zweier ehemaliger Ostblockstaaten - der DDR und Rumänien - und ihren Geheimdiensten -Stasi (Staatssicherheitsdienst) und Securitate - vor.

Die Aufarbeitung der kommunistischen Vergangenheit bedeutete eine große Herausforderung für Prozesse des nationalen wie transnationalen Erinnerns. Gerade die ,asymmetrische Beziehung extremer Gewalteinwirkungen," wie Aleida Assmann den stalinistischen Terror nennt, der in diesen Ländern herrschte, muss in den Mittelpunkt rücken. Dazu bedarf es, so Assmann, zwischen Tätern und Opfern eines „Erinnerungsvertrag[es]“, bevor das von den Einen verübte und den Anderen erlebte Trauma verarbeitet werden kann, um eine Grundlage für eine gemeinsame Zukunft legen zu können (Assmann, Gewalt, 45-46). Eine solche Zukunft ist notwendig für die Befriedung und Aussöhnung der Gesellschaft, damit sie nicht in einem endlosen Teufelskreis der Gewalt endet. Assmanns „Erinnerungsvertrag“ erkennt das Leid der Opfer ,,auf der Basis der Menschenrechte“ an und zollt sowohl den ermordeten als auch den überlebenden Opfern die Anerkennung, dass ihre Zeugenschaft als Teil der Erinnerungskultur sowohl der Opfer als auch der Täter angenommen wird. So ist das Erinnern auf die Zukunft ausgerichtet und kämpft gegen das Vergessen einer traumatischen Vergangenheit (Assmann, Gewalt, 45).

Die offene und öffentliche Aufarbeitung der Vergangenheit wurde in nahezu allen Staaten des ehemaligen Ostblocks angestrebt, als man seit den neunziger Jahren Kommissionen und Behörden einrichtete, die den Umgang mit den Akten der ehemaligen Geheimdienste normieren sollten. Diese Institutionen leisten auch nach mehr als fünfundzwanzig Jahren seit dem Ende des Kalten Krieges einen wichtigen Beitrag zur Aufarbeitung der Vergangenheit. Auf der einen Seite organisieren sie die Erfassung der Unterlagen der ehemaligen Geheimdienste und regeln den Umgang mit diesen Akten, so dass die Einflussnahme der Staatssicherheitsdienste auf Individuen oder Gruppen und deren Schicksal aufgeklärt werden kann. ${ }^{1}$ Auf der anderen Seite fördern sie eine neue Erinnerungskultur, indem sie Betroffenen, Tätern aber auch Forschern Zugang zu den Akten gewähren. So kann jede Akte aus mehreren Perspektiven betrachtet werden, die einander ergänzen und ein mehrstimmiges Erinnern ermöglichen. Aleida Assmann nennt diese Art mit der Vergangenheit umzugehen „dialogisches Erinnern“ und definiert sie als eine Art des historischen Erinnerns, in dem die Geschichte aus verschiedenen Perspektiven, nicht nur aus den drei „,sanktionierten“ - die der Täter, der Opfer oder der Märtyrer-, und im Dialog miteinander betrachtet wird (Assmann, Gewalt, 48). So entsteht, laut Assmann, ,die wechselseitige Verknüpfung und Aufrasterung allzu einheitlicher Gedächtniskonstruktionen“ (Assmann, Gewalt, 48), die dazu führt, dass das Erinnern an eine traumatische Zeit, nicht nur in schwarz oder weiß oder schwarz-weiß gepflegt wird, sondern auch die verschiedenen grauen Stellen ans Licht gebracht werden. In Bezug auf den mehr als vierzig Jahre andauernden Kommunismus in Osteuropa ermöglicht 
das dialogische Erinnern den Austausch nicht nur zwischen Tätern und Opfern, sondern bietet auch zwiespältigen Figuren - offiziellen oder inoffiziellen Mitarbeitern eines Geheimdienstes oder Kollaborateuren eines Regimes - die Gelegenheit, sich zu Wort zu melden.

Ausgangspunkt der oben erwähnten Behörden und Kommissionen waren die hinterlassenen Archive der Geheimdienste. "The history of the archive is the recognition of loss", behauptet Peter Fritzsche in seiner Studie zur Institution des Archivs im deutschen Kontext (187). Die Beweggründe für die Etablierung von Archiven sind in der Regel die Bewahrung vor dem Verlust und Vergessen. Wie Jacques Derrida uns in seinem bahnbrechenden Essay „Archive Fever“, erinnert, geht mit der Verwaltung des Archivs auch Machtausübung und die Übernahme von Verantwortung einher: "[t]here is no political power without the control of the archive, if not of memory" (11). Nach dem Fall des Kommunismus ermöglichte in diesem Sinne die Öffnung der Archive der osteuropäischen Geheimdienste Forschern wie auch Betroffenen Einsicht in diese Apparate, ihre Strukturen und Arbeitsmethoden. Aber, wie Derrida deutlich macht, Archive zu beherbergen, bedeutet, sie auch zu verbergen, da die Überleitung von einer Institution zu einer anderen und der Übergang vom privaten zum öffentlichen Gebrauch nicht immer den Wandel "from the secret to the nonsecret" (10) bedeutet. Die neuen ArchivarInnen regeln den Zugang zu den Akten und Dokumenten der alten Machthaber ebenso wie die neu entstandenen Geheimdienste den Zugang zu den Akten der nachfolgenden Regierungen regulieren. Wie der "Historiker" in Carolyn Steedmans Studie Dust: The Archive and Cultural History, ist der Forscher oftmals in den Archiven ein unbeabsichtigter Leser, "[who] will always read that which was never intended for his or her eyes" (75).

Die Bestände in den jeweiligen Archiven enthüllen das Ausmaß der Überwachungstätigkeiten dieser Geheimdienste, wie auch ihre Sichtweise auf die Gesellschaft, die streng geheim bleiben sollte. Die ursprünglich akribisch angelegten und archivierten Akten dieser inzwischen untergegangenen geheimpolizeilichen Verwaltungen demonstrieren, wie sehr diese modernen Geheimdienste dazu beitrugen, Angst und Misstrauen in der Bevölkerung zu verbreiten und zu fokussieren. Wie Fiona Capp erläutert, war es der dubiose Verdienst aller Geheimdienste im Kalten Krieg, zwischenmenschliche Beziehungen - im Westen waren es von Kommunisten, im Osten von Dissidenten oder Andersdenkenden - als Ausdruck gezielter, feindlicher, politischer Tätigkeit oder noch schlimmer eines politischen Netzwerks zu interpretieren (Capp 10). Auf diese Weise nahm der Staatsfeind ,mythic proportions as an imagined enemy, a one-dimensional creature whose individual complexity is denied" an (10-11). Geheimdienste im Kalten Krieg waren einem vereinfachten Freund-Feind-Schema verhaftet, das für die ideologische Kriegsführung auf beiden Seiten kennzeichnend war. Nach Bernd Stöver war der Kalte Krieg ,,auch eine innergesellschaftliche Auseinander- 
setzung mit den angeblichen oder tatsächlichen Parteigängern des jeweils anderen Lagers“, eine Auseinandersetzung, welche ,jeweils unterschiedlich ausgeprägt, aber immer präsent war" (Stöver 227). Antrieb der flächendeckenden Überwachungsmaßnahmen von Geheimdiensten totalitärer Prägung waren besonders chronische Verfolgungsängste und ungezügelte Verschwörungstheorien, die interne Dissens nur über das stark polarisierte Freund-FeindSchema interpretierte und auch ahndete. Dabei imitierten die Sicherheitsapparate auf Staatsseite das Verhalten der angeblichen ,Verschwörer', die sie imaginierten, womit sie letzten Endes zur Ausbreitung von Fantasien von Verschwörern und einem vom Feind gefährdeten Nationalstaat beisteuerten.

Die Bestände totalitärer Geheimdienste zeigen überdies ihre eigene Ordnung der Dinge, genauer des hinterlassenen Beweismaterials (Informantenberichte, Lageberichte, Einschätzungen zu Personen, Photographien, Aufnahmen und Aufzeichnungen von abgehörten Gesprächen und Verhören usw.). Wissenschaftler stehen vor der Aufgabe, aus der überwältigenden Menge an Akten die leitenden Organisationsprinzipien dieser geheimen ArchivarInnen herauszudestillieren, vor allem im Wissen, dass trotz objektivierbaren Prinzipien der damaligen Archivierung zufällig nur diese Dossiers ad acta gelegt wurden, während andere aus heute unerfindlichen Gründen zerstört wurden. So umfassend die Archivbestände auch immer sind, sie sind nicht lückenlos, wie Steedman uns erinnert: "[t]he Archive is made from selected and consciously chosen documentation from the past and also from the mad fragmentation that no one intended to preserve and that just ended up there" (68). Im Fall von Geheimdienstdokumenten stehen wir als WissenschaftlerInnen vor einem Paradox, dass einerseits Unmengen von Material als Beweis für vermeintlich staatsfeindliche Tätigkeiten sorgfältigst gesammelt, vervielfältigt und archiviert wurden, andererseits dass das Material notwendigerweise fragmentiert bleibt.

Das Ministerium für Staatssicherheit, das auf die „flächendeckende“ Überwachung der eigenen Bevölkerung in der DDR abzielte und 116 Aktenkilometer anlegte, war Spitzenreiter unter den Ostblockregimes, insbesondere im Vergleich zu seinem berüchtigten rumänischen Gegenstück, der Securitate, die es ,nur" auf 25 Aktenkilometer brachte. Beide hinterließen Opfer-, Informanten- und Kaderakten, aber auch Dokumente, die konspirative Vorgänge gegen Personen und Gruppen, Festnahmen, politische Prozesse und Transkripte von abgehörten Gesprächen betreffen. Im Fall von bekannten Dissidenten oder denjenigen Autoren, die unter dem Verdacht standen, kritische oder staatsfeindliche Werke verfassen und publizieren zu wollen, haben die Geheimdienste durch ihre Wachsamkeit ebenfalls als Archiv gedient für zahlreiche unveröffentlichte Manuskripte, Briefe, Liedertexte und Schriften anderer Art. Bei den rumäniendeutschen Schriftstellern Herta Müller und Richard Wagner ging die ,Betreuung' der Securitate sogar soweit, dass die Geheimpolizei die deutschsprachige Literaturzeitschrift Neue Literatur abon- 
nierte, um ihre literarischen Aktivitäten besser überwachen zu können (ACNSAS, 152).

Unter den hinterlassenen Dokumenten der Stasi, die sich in vielen Opfer- und Informantenakten finden, sind unter anderem Berichte zu Personen und literarischen Werken zu finden, die Opfer der Zensur wurden. Neben dem Stasi-Unterlagen-Archiv (BStU) ist das ergiebigste Archiv für die Erforschung von Zensurmechanismen das des ehemaligen Ministeriums für Kultur (MfK). Das MfK beherbergte die ,höchste“ Zensurinstanz der DDR (Costabile-Heming 56), die Hauptverwaltung Verlage und Buchhandel, die aber nie im Alleingang agierte, sondern in den meisten Fällen in Zusammenarbeit mit anderen „Akteuren“ der Zensur wie den Lektoren der Verlage, Ministern und Politbüromitgliedern und letztlich auch der Stasi handelte. ${ }^{2}$ In Ostblockdiktaturen erschöpfte sich die Zensur nur in seltenen Fällen in schlichten und kategorischen Verboten. In der DDR zum Beispiel regelte der Staat alle Phasen der Produktion von Literatur, angefangen mit der Vorzensur während der Konzipierung von Buchprojekten über die Verfassung oder Fertigstellung von Manuskripten bis hin zu der Nachzensur in Form der akribischen Beobachtung und gelegentlichen Verfolgung von unliebsamen Autoren und deren Werken. Im Anbetracht der Spannbreite der Zensurmechanismen, bzw. des gesamten Zensurdispositifs, war Zensur in der DDR häufig das Resultat von komplexen und oft lange hingezogenen Verhandlungen zwischen den jeweiligen sozialen und politischen Akteuren. Sie war außerdem immer ein fester Bestandteil der diskursiven Produktion von literarischer Kommunikation, wie Sophia Rosenfeld behauptet (129), und somit, wie Judith Butler anmerkt, eine „,bildende“ Komponente sowohl des literarischen wie auch des politischen Feldes (252). Die Archive der kommunistischen Regime vermögen daher faszinierende Einblicke in die höchst umkämpfte Zone zu gewähren, in der Literatur und Politik sich trafen und überschnitten. Sie werfen ein Schlaglicht nicht nur auf die jeweiligen Entscheidungen von Individuen und Institutionen sowie deren Habitus und Dispositionen, sondern auch auf ihre unterschiedlichen Interventionen oder Stellungnahmen im literarischen Feld. ${ }^{3}$

Die Beiträge in diesem Heft gehen von Assmanns Konzept des ,,dialogischen Erinnerns" aus und bieten Beispiele von Dialogen zwischen Opfern und ihren archivalischen ,Schatten', vermittelt durch Texte und Performance (Glajar und Garde), Tätern und ihren Akten im Dokumentarfilm (Lewis) und zwischen Zeitzeugen und der eigenen erinnerten Geschichte in Übersetzungen (Kind) sowie in einem autobiographischen Roman (Petrescu) an. Valentina Glajar kontrastiert in ihrem Beitrag das Werk Cristina und ihre Attrappe oder Was (nicht) in den Akten der Securitate steht (2008) mit der von der Securitate angelegten Akte Herta Müllers. Während Müller in ihrer Schrift hauptsächlich auf die Lücken in der Akte verweist, konzentriert sich Glajar auf das, was tatsächlich in der Akte steht. So sprechen die beiden Texte zueinander, und Glajars aufmerksame und detailorientierte Leseart entlarvt subtile Unter- 
schiede zwischen ihnen. Dabei gelingt es ihr, einige der von Müller beklagten Leerstellen zu füllen, indem sie Informationen nicht nur aus Müllers, sondern auch aus der Akte ihres damaligen Ehemannes Richard Wagner heranzieht. Besonders wertvoll sind für sie die Aufzeichnungen der abgehörten Gespräche der zwei Schriftsteller, die sie, wenn auch vermittelt, durch die Transkription der Securitate zu Wort kommen lassen. Diese kontrastive Leseart erlaubt es Glajar, eine nuanciertere Aktengeschichte anzubieten.

Ulrike Gardes Beitrag widmet sich zwei zeitgenössischen Theaterproduktionen des Künstlers Clemens Bechtel, Meine Akte und ich (2013) und Staats-Sicherheiten (2008), in denen es um eine Auseinandersetzung mit den Unterlagen der ehemaligen Stasi geht. Um diese Unterlagen auf die Bühne zu bringen und dadurch ein Gespräch zwischen Opfern, Tätern und der zeitgenössischen deutschen Gesellschaft anzuregen, entschied sich Bechtel, keine Schauspieler, sondern die in den Akten erwähnten ehemaligen DDR-Bürger zu Wort kommen zu lassen. Diese von Garde und Meg Mumford als „Theatre of Real People" bezeichnete Performance-Art bringt eine neue Betrachtungsweise der problematischen Vergangenheit der DDR hervor, die als Ausgangspunkt für eine neue und produktive Beschäftigung mit der Erinnerungskultur der DDR dienen kann.

Alison Lewis befasst sich mit Annekatrin Hendels Dokumentarfilm Vaterlandsverräter (2012), der die IM-Tätigkeit des Schriftstellers Paul Gratzik thematisiert. Während Stasi-Akten generell als zuverlässig betrachtet werden, weil sie die Bedeutung von "testimonial objects" annehmen, beweist Lewis in ihrer Analyse, dass die Performanz von Geständnissen von StasiMitarbeitern, wenn auch nicht den Akten entsprechend, immerhin einen konstruktiven Beitrag zur Bewältigung der DDR-Vergangenheit beisteuern kann. Lewis zeigt, dass im Unterschied zu anderen Werken, die sich dem Thema Stasi-Mitarbeit nähern, Hendel in ihrem Film nicht nur den Stasi-Unterlagen, die in der BStU zu finden sind, sondern auch Gratziks Erinnerungen an seine Zeit als IM Gehör schenkt. Hendel benutzt die Stasi Unterlagen und die ihnen zugeschriebene Autorität nicht, um die „Wahrheit“ über Gratzik zu entlarven, sondern um die Vergangenheit und die Gegenwart in Bezug auf einander zu untersuchen und sie miteinander sprechen zu lassen. Durch dieses zweifach dialogische Verfahren zeigt Hendel eine Möglichkeit zur Aussöhnung zwischen Opfern und Tätern des ehemaligen DDR-Regimes auf.

Der Aufbau Verlag veröffentlichte zu Zeiten der DDR die Werke des berühmtesten portugiesischen Vertreters des Realismus José Maria Eça de Queirós (1845-1900) in deutscher Übersetzung. Anette Kind analysiert in ihrem Artikel, wie diese Übersetzungen zustande kamen und welchen Bedingungen sie entsprechen mussten, um gedruckt zu werden. Da Eça de Queirós dank seiner kreativen Wortschöpfungen und seines ausgefeilten Stils im Portugiesischen als Erneuerer der literarischen Ausdrucksmöglichkeiten gilt, interessiert es Kind auch, inwieweit sein Stil in den deutschen Übersetzungen 
des Aufbau Verlags beibehalten wurde. Ihre Recherchen im Verlagsarchiv, ergänzt durch ihre Gespräche mit Zeitzeugen und die Analyse unterschiedlicher Übersetzungen desselben Textes zu verschiedenen Zeiten der DDR, beweisen, dass die für die DDR üblichen Zensurmechanismen auch für literarische Übersetzungen galten.

Corina L. Petrescus Artikel befasst sich mit dem Roman Rote Handschuhe (2001) des siebenbürgisch-sächsischen Schriftstellers Eginald Schlattner (1933), der seit seiner Veröffentlichung für Kontroversen innerhalb der rumäniendeutschen Landsmannschaft gesorgt hat. Der Roman thematisiert die Haftzeit und letztendliche Zusammenarbeit eines stark autobiographischgeprägten Ich-Erzählers mit der Securitate am Ende der 50er Jahre. Der Autor stellt sich seinen Erinnerungen und meint, ein Werk geschrieben zu haben, das zur Versöhnung mit denen, die wegen seiner Aussagen während der Haft gelitten haben, führt. Jedoch hat der Roman alles andere als eine Aussöhnung der Beteiligten herbeigeführt. In ihrer Auseinandersetzung mit dem Werk fragt sich Petrescu, inwieweit der Roman auch tatsächlich ein Mea culpa oder Schuldbekenntnis ist. Ihre stilistische Analyse ergibt, dass, auch wenn der Roman zur Diskussion um die Bewältigung der rumäniendeutschen Vergangenheit beiträgt, er letzten Endes den Sinn eines Mea culpa verfehlt.

Wie alle Beiträge in diesem Themenheft nahelegen, ist eine dynamische und kritische Erinnerungskultur für die Aufarbeitung von Gewalt und Schuld vonnöten. Erinnerungsarbeit der kommunistischen Vergangenheit muss sich sowohl der Autorität des Archivs als auch der individuellen Zeugenschaft bedienen, denn, wie Assmann behauptet, sind Geschichte und Gedächtnis längst „keine Rivalen“ mehr (Assmann, History, 261). Für die WissenschaftlerInnen jedoch bergen beide Quellenarten eine Herausforderung, denn das menschliche Gedächtnis, so wichtig es auch ist, um persönliche Erfahrungen von Verfolgung und Repression zu dokumentieren, ist von Natur her oft selektiv und unzuverlässig. So bedeutsam der Umgang mit den Archiven der Geheimdienste auch ist, birgt er gleichermaßen seine Schwierigkeiten. Mit der Öffnung der Akten kommt vor allem Verantwortung, aber auch eine gewisse Macht mit der Verwaltung der Akten und der Regelung des Zugangs dazu. Obwohl in Rumänien wie in Deutschland die Zukunft des Archivs noch gesichert zu sein scheint, liegt das postkommunistische Rumänien, das die Archive viel später geöffnet hat, in Vergleich zu Deutschland noch weit zurück. Auch wenn die Aufarbeitung der Vergangenheit, die sowohl den Erfahrungen von Opfern wie auch Tätern Rechnung trägt, in vielen Fällen eine utopische Vorstellung ist, ist sie ein Ideal, worauf diese Nachfolgegesellschaften nicht verzichten können. Vor allem solange Opfer wie auch Täter noch am Leben sind, braucht die Aufarbeitung der Vergangenheit das kommunikative Gedächtnis von Zeitzeugen, damit dieses ein kulturelles Gedächtnis werden kann, und zwar eines, das von allen Gruppierungen anerkannt und geteilt wird. Paul Gready hat überzeugend behauptet, dass Kultur eine Rolle 
zu spielen hat ,,in [the] process of democratic thickening and re-imagining [of a society since] [i]t can keep sniping at the shortcomings of the past and present [and] ask the difficult questions" (47). Dieses Themenheft möchte dazu beitragen, die Bedeutung von Kultur im Prozess der postkommunistischen Aussöhnung auf individueller und kollektiver Ebene hervorzuheben.

${ }^{1}$ Siehe dazu z. B. das "Stasi-Unterlagen-Gesetz", das die Aufgaben des Bundesbeauftragten für die Stasi-Unterlagen regelt: http://www.bstu.bund.de/DE/BundesbeauftragterUnd Behoerde/Rechtsgrundlagen/StUG/stug_node.html und die Grundsätze des Landesrates für das Studium der Securitate-Archive (CNSAS), der in Bukarest den Umgang mit den Akten der Securitate regelt: http://www.cnsas.ro/cadrul_legal.html. Beide abgerufen am 30.08.2017.

${ }^{2}$ Der Zensurapparat umfasste fünf getrennte Komplexe - die Verlage, die SED, die Hauptverwaltung Verlage und Buchhandel im Ministerium für Kultur, das Büro für Urheberrechte, ebenfalls im Kultusministerium und zuletzt, als fünfter Zensor, die Stasi. Siehe Walther 131.

${ }^{3}$ Siehe Stephen Parkers und Matthew Philpotts ‘ Anwendung der soziologischen Theorien Pierre Bourdieus auf das literarische Feld in der DDR, das ihnen zufolge durch fundamentale Gegensätze strukturiert war: einerseits durch den ,autonomen " und von der Politik am wenigsten beeinflussten Pol, andererseits durch den ,heteronomen“ Pol, der von der Politik (SED) am stärksten kontrolliert wurde. Parker und Philpotts 5.

\section{Zitierte Literatur}

ACNSAS (Arhiva Consiliul Naţional pentru Studierea Arhivelor Securităţii/Archiv des Landesrates für das Studium der Securitate-Archive). FI, Akte 184945 (Richard Wagner).

Assmann, Aleida. ,History, Memory, and the Genre of Testimony.“ Poetics Today 27.2 (2006): 261-273.

—. , ,Von kollektiver Gewalt zu gemeinsamer Zukunft: Vier Modelle für den Umgang mit traumatischer Vergangenheit." Kriegserfahrung und nationale Identität in Europa nach 1945. Erinnerung, Säuberungsprozesse und nationales Gedächtnis. Kerstin von Lingen (Hg.). Paderborn: Schöningh, 2009. 42-51.

Butler, Judith. „Ruled Out. Vocabularies of the Censor.“ Censorship and Silencing: Practices of Cultural Regulation. Robert C. Post (Hg.). Los Angeles: Getty Research Institute 1998. 247-259.

Capp, Fiona. Writers Defiled. Security Surveillance of Australian Authors and Intellectuals 1920-1960. South Yarra: McPhee Gribble, 1993.

Costabile-Heming, Carol Anne. ,'Rezensur'. A Case Study of Censorship and Programmatic Reception in the GDR." Monatshefte 92.1 (2000): 53-67.

Derrida, Jaques. „Archive Fever. A Freudian Impression.“ Translated by Eric Prenowitz. Diacritics 25.2 (Summer 1995): 9-63.

Fritzsche, Peter. ,The Archive and the Case of the German Nation." Archive Stories: Fact, Fiction, and the Writing of History. Antoinette Burton (Hg.). Durham, NC: Duke, 2005. 184-209.

Gready, Paul. „Culture, Testimony, and the Toolbox of Transitional Justice.“ Peace Review: A Journal of Social Justice 20.1 (2008): 41-48.

Parker, Stephen und Matthew Philpotts. ,Sinn und Form“. The Anatomy of a Literary Journal. Berlin: de Gruyter, 2009.

Rosenfeld, Sophia. „Writing the History of Censorship in the Age of Enlightenment." PostModernism and the Enlightenment. New Perspectives in French Intellectual History. Daniel Gordon (Hg.). New Haven: Yale UP, 2001. 
Steedman, Carolyn. Dust. The Archive and Cultural History. New Brunswick, NJ: Rutgers UP, 2002.

Stöver, Bernd. Der Kalte Krieg. Geschichte eines radikalen Zeitalters 1947-1991. München: C. H. Beck, 2007.

Walther, Joachim. „Der fünfte Zensor. das MfS als die letzte Instanz.“ Zensur im modernen deutschen Kulturraum. Beate Müller (Hg.). Tübingen: Niemeyer, 2003. 131-148. 